\title{
Integral-based Material Point Method and Peridynamics Model For Animating Elastoplastic Material ${ }^{\star}$
}

\author{
Yao Lyu ${ }^{1}$, Jinglu Zhang ${ }^{1}$ Ari Sarafopoulos ${ }^{1}$, Jian Chang ${ }^{1}$, Shihui Guo ${ }^{2}$, and \\ Jian Jun Zhang ${ }^{1}$ \\ 1 National Centre for Computer Animation, Bournemouth University, Dorset, UK \\ \{ylyu, zhangj, asarafop, jchang, jzhang\}@bournemouth.ac.uk \\ 2 School of Software, Xiamen University, Xiamen, China \\ guoshihui@xmu.edu.cn
}

\begin{abstract}
This paper exploits the use of Material Point Method (MPM) for graphical animation of elastoplastic materials and fracture. Previous partial derivative based MPM studies face challenges of underlying instability issues of particle distribution and the complexity of modeling discontinuities. This paper incorporates the state-based peridynamics structure with the MPM to alleviate these problems, which outweighs differential-based methods in both accuracy and stability. The deviatoric flow theory and a simple yield function are incorporated to animate plasticity. To model viscoelastic material, the constitutive model is developed with the linearized peridynamics theory which regards the current configuration as equilibrated and is only influenced by current incremental deformation. The peridynamics theory doesn't involve the deformation gradient, thus it is straightforward to handle the problem of cracking in our hybrid framework. To ease the implementation of the fracture divergence under MPM, two time integration methods are adopted to update the crack interface and continuous parts separately. Our work can create a wide range of material phenomenon including elasticity, plasticity, viscoelasticity and fracture. Our framework provides an attractive method for producing a variety of elastoplastic materials and fracture with visual realism and high stability.
\end{abstract}

Keywords: Material Point Method · Peridynamics · Elastoplastic modeling · Computer Animation.

\section{Introduction}

Physically-based modeling of elastoplastic material has been an active research topic for many years in computer graphics, particularly for its appealing application in visual effects industry. Scenes involving elastoplastic deformation are

\footnotetext{
* This work is supported by National Natural Science Foundation of China (61702433, 61661146002), the Fundamental Research Funds for the Central Universities, VISTA AR project (funded by the Interreg France (Channel) England, ERDF), the China Scholarship Council and Bournemouth University
} 
very common and varied, for example, clothes moving with wind, rubber toys bouncing on the floor, flowing honey, or the broken plastic board. In order to model such realistic behaviors under different circumstances, the robust simulation method needs to be capable of handling complex topological changes and various contact responses, such as collision and cohesion. To find the simulation method that can naturally model elastoplastic material along with complex topological changes is the current focus of the field.

Meshless simulation methods are powerful in dealing with complicated topological changes since it does not require high quality mesh and efforts to overcome the issues from severe mesh distortion. The MPM [29][30] is an extension of the particle-in-cell (PIC) method. It combines the Eulerian Cartesian grids and Lagrangian particles: a continuum body is discretized into a set of particles, also referred to as material points that are free to move on top of the background Eulerian grid [16]. The grid uses partial derivatives of particles' displacement to solve the governing equations. The MPM can naturally process material point distribution and self-collisions. It also has been proved to be especially suitable for animating materials that undergo large deformations [11]. Despite its physical realism and geometrical convenience, the traditional MPM solver has several disadvantages: 1) Due to the governing equation based on spatial derivatives of displacements, the results are sensitive to the underlying particle distribution [7]. Also it has difficulty in solving singularity along discontinuities. 2) To observe boundary details, MPM has to maintain a fine resolution grid which brings high computational costs for particle-grid transfer, particularly throughout the whole simulation domain. While researchers have extensively studied refining regions of particular interest by using an adaptive grid [5], the ability to simulate detailed discontinuities dynamics, such as crack propagation, is still limited.

Recently, peridynamics has gained its popularity in meshless simulation for discontinuous deformation, originally proposed by Silling [20]. Peridynamics theory defines that a point in a continuum interacts directly with other points separated from it within a finite distance. The advantage of the peridynamics is that its way of treating the discontinuous parts, which may appear in the continuum body as a result of the strain or stress, is exactly the same as continuous part. The linear peridynamics treats the deformation more generally by processing the small deformation based on the referenced configuration, which provides some possibilities to simulate the materials with both solid and fluid properties. Due to its particle-based nature, peridynamics needs the additional efforts to handle complex physical contacts. Furthermore, the peridynamics originates from the solid mechanics, focusing on mechanical experiments. Currently there are only a few mature models and experiments in continuum mechanics being adopted by peridynamics methods for animating elastoplastic material [34].

A preliminary version of this work has been reported which created the innovative MPM with peridynamics truss particle structure for modeling several kinds of elastoplastic objects [15]. This work continues to investigate the concept of integrating the deformation displacement and simulation the response, while 
introducing the linearized peridynamics theory to create more general effects, such as viscoelastic solids and fluids. We present four main contributions:

Elasticity We equip material point with virtual bonds and family points. The elastic energy density function is redefined in an integral way with this truss structure. Varied stiffness of elastic materials can be simulated with high realism and stability.

Plasticity The virtual bond structure makes our model trivial to model plastic behaviors. We use a novel method to extract plasticity from the deviatoric part of constitutive model and accumulate plastic increment permanently at particle-grid transfer step.

Viscoelasticity We incorporate a general peridynamics model to process the dynamics of viscoelastic materials using the modulus state. The force density is determined by the incremental deformation of bonds in the horizon which is suitable for the situation undergoing large deformation.

Fracture We handle crack definition and propagation through deactivating the virtual bonds. The fracture criterion of each bond involves the deformation status of the connected material points and the grid cell it stays. We update fracture surfaces and fracture inner parts in different time integration methods. Our method avoids the difficulty of duplicating grids and large computation cost brought by multiple particle-grid transfer processes.

After discussing related work in Section 2, we outline the methodology and explain the hybrid particle-grid structure in Section 3. Section 4 describes the constitutive model for modeling elastoplastic material in details. The governing equation and the constitutive model of viscoelastic materials are explained in Section 5. Crack definition and propagation are discussed in Section 6. Experimental examples for evaluating our method and discussion of results are given in Section 7. In Section 7 we conclude the method and explain our future work.

\section{Related Work}

Elastoplastic modeling Terzopoulos and Fleischer [32][33] pioneered the elastic and plastic simulation methods in computer graphics. O'Brien and colleagues [17] incorporated the finite element method (FEM) with multiplicative plasticity model and obtained realistic motion for a much wider range of materials. Later, Gerszewski et al. [6] adopted the deformation gradient for animating elastic behaviors based on point method. Levin [12] rediscretized elasticity on a Eulerian grid, which is similar to MPM grid. Based on previous hybrid grids and particle modeling methods, Stomakhin [27] incorporated energetically consistent invertible elasticity models into MPM for modeling snow, varying phase effects. Daniel and his colleagues [18] avoided computating the SVD in Stomakhin's work and used semi-implicit MPM discretization of viscoelasticity allowing for high spatial resolution simulations. They achieved a wide range of viscoelastic, complex fluid effects. Recently, Chen [4] presented a novel elastoplastic constitutive model to handle brittle fracture and ductile fracture in the peridynamics-based framework. At the same time, many researchers focused on developing the real time 
and haptic simulator for the elastic materials, such as garments. For example, Salsedo et al. [19] designed the HAPTEX system using dynamically variable spatial resolution to reduce the computational burden during rendering the fabrics, which Bottcher [1][2] extended by implementing separated computation threads for different simulation scales.

Material Point Method MPM is a hybrid grid-particle method using the Cartesian grid to resolve topology changes, treat self-collisions and fracture naturally. It tracked mass, momentum dynamics and particle deformation through combining the Lagrangian theory without the inherent need for Lagrangian mesh connectivity, devised by Sulsky [29][30] for engineering applications. Stomakhin et al. [27] introduced MPM into computer graphics. They tracked the deformation gradient on particles and transformed the deformation back to grid nodes. This work obtained a wide variety of snow phenomenon. Later, Jiang and his colleagues proved that MPM is a useful method for granular materials by animating sand [10]. Based on previous work of granular materials, Tampubolon extended the MPM to simulate multi-phase behaviors through using multiple grids, such as porous sand and water interactions [31]. A majority of elastoplastic MPM works for computer graphics [9][28] focus on resolving intensive collision scenarios on the surface or the curve with millions degrees of freedom. Unlike above studies, our method incorporates the integral-based constitutive model to replace the typical partial derivative based model. This helps us alleviate the instability and difficulty issues from particle distribution of arbitrary elastoplastic deformation. For MPM, the final resolution highly depends on the grid resolution which means high quality simulation results in increased computation. This is remedied by our particle truss construction and the bond structure. With virtual bonds, the constitutive model can demonstrate detailed topological changes smaller than the grid cell.

Peridynamics The peridynamics is an extension of solid mechanics method in which points in a continuum interact with nearby points. Different from Smoothed Particle Hydrodynamics (SPH), peridynamics is a non-local method. Instead of using spatial differential formulations, it uses spatial integral equations as the governing equations. Silling proposed the peridynamics theory [20] for efficiently and uniformly solving problems involving both continuities and discontinuities [24][4][7]. Its application sparked the engineering applications, such as multiscale material modeling [25] and crack dynamics [23]. Levine [13] introduced the peridynamics theory to computer graphics. He revisited brittle fracture studies by characterizing peridynamics as spring-mass systems with two specific parameters, strain metric and interaction horizon. Silling [21] proposes the linear version of peridynamics theory to simulate more general materials, such as linear fluid and viscoelastic objects. This method provided the possibility of multi-scale application using peridynamics [22]. Currently, most research [4][7] is focused on how to reformulate elastic constitutive models and produce persuasive effects. However, the theoretical equivalence of peridynamics com- 
pared to continuum mechanics remains unclear [34]. A lot of mature theories and experiments in continuum mechanics have not been adopted for peridynamics in computer graphics. This motivates us to define the integral-based constitutive model equipped with the peridynamics structure within the MPM framework for simulating versatile elastoplastic materials.

\section{Method}

The governing equation of MPM arises from basic conservation of mass and momentum [27]. Weak formulation is obtained by multiplying the balance of momentum and integrating the governing equation over initial volume, we therefore propose an integral force density function $F^{s}\left(x_{p}\right)$ to replace spatial derivatives of displacement and redefine the weak formulation as:

$$
\int_{\Omega} \rho a_{p} \delta u_{p} d \Omega+\int_{\Omega} \rho F^{s}\left(x_{p}\right) \delta u_{p} d \Omega=\int_{\Omega} \rho b_{p} \delta u_{p} d \Omega+\int_{\Gamma_{\tau}} \rho \delta u_{p} \overline{\tau_{p}} d \Gamma
$$

where $\Omega$ denotes the integrating region in the current configuration, $\rho$ is density, $a_{p}$ is the acceleration of particle $\mathrm{p}, \delta u_{p}$ is the virtual displacement (infinitesimal feasible changes where constraints remain satisfied). $b_{p}$ is the body force, for example, gravity. $\tau_{p}$ is the surface traction on part of the boundary $\Gamma_{\tau}$.

This paper adopts the grid-particle transfer procedure of the MPM, however, with different way of collecting the deformation of particles. In our MPM framework, the material domain at $t^{n}$ is discretised with particles at $x_{p}^{n}$. Each particle has volume $V$, mass $m_{p}$, velocity $v_{p}^{n}$, and other physical quantities, such as deformation matrix $F_{p}$, Lamé parameters $\mu_{p}$ and $\lambda_{p}$, plastic yield parameters $\psi_{p}$. In each time step, a new grid is generated. Grid node $I$ is used to store nodal parameters, such as position $x_{I}$, mass $m_{I}$, velocity $v_{I}$, force $f_{I}$. Our framework adopts dyadic products of one-dimensional cubic B-splines as a basic weight function in [27] during the particle-grid transfer process. Here we outline the full update procedure:

1)Particle-to-grid transfer Transfer material point mass $m_{p}$ and momentum $(m v)_{p}$ to the grid nodes as $m_{I}$ and $(m v)_{I}$.

2)Compute internal forces The internal force of grid node $I$ is calculated based on the stress tensor of each point, presented as $f_{I}^{I N T}=\sum_{N p} m_{p} N_{I}\left(x_{p}\right) F^{s}\left(x_{p}\right) V$. This equation is derived from our integral-based energy density function. The detailed description of the update rule for $F^{s}\left(x_{p}\right)$ is given in Section 4 .

3)Update Grid Momentum Nodal velocities at the timestep $n+1$ are updated by $\widehat{v}_{I}^{n+1}=v_{I}^{n}+\triangle t f_{I} / m_{I}^{n}$ for explicit time integration. $f_{I}$ is the total force.

4)Grid-based body collisions Grid velocity $v_{I}^{n+1}$ is updated by the collision field and friction parameter from [27].

5)Grid-to-particle transfer Transfers updated nodal velocity $v_{I}^{n+1}$ and momentum $(m v)_{I}^{n+1}$ to particles. 
6)Particle collisions Modify $v_{p}^{n+1}$ by collision field on particle level to obtain detailed deformation behaviors, especially on the boundary.

7)Fracture Based on the current particle distribution and the deformation status of the grid cell, we remove the virtual bond that intersects with the fracture plane. The fracture model is discussed in Section 6 .

\section{Elastoplastic Models}

In contrast to the partial differential equations used in the classical formulations, here we describe the internal force density function using an integral formulation of pairwise bond forces, inspired by Silling's constitutive model [24]:

$$
F^{s}\left(x_{p}\right)=\int_{H_{x_{p}}}\left[T<x_{p}, x_{p}{ }^{\prime}>-T<x_{p}{ }^{\prime}, x_{p}>\right] / \rho d H_{x_{p}}
$$

$x_{p}{ }^{\prime}$ is the neighbor point of point $x_{p}$. Our work is motivated by the peridynamics theory in which a virtual bond is built between each family point pair. It is called as truss structure: each neighbor material point $x_{p}{ }^{\prime}$ of material point $x_{p}$ are referred to as its family members $H_{x_{p}} . x_{p}$ has interaction with all its family members at same time. Each interaction is operated by a virtual bond. $T<x_{p}, x_{p}^{\prime}>$ represents the interaction force between $x_{p}$ and $x_{p}{ }^{\prime}$. The $F^{s}\left(x_{p}\right)$ collects the particle deformation in an integral way, avoiding the use of using the spatial derivatives of displacements $\frac{\delta u_{x_{p}}}{\delta x_{p}}$.

\subsection{Elastic Model}

When the elasticity is the only consideration, the energy density function is defined in [26] as:

$$
E^{s}=\mu\left\|F_{e}-R_{e}\right\|^{2}+\frac{\lambda}{2}\left(J_{e}-1\right)^{2}
$$

where $F_{e}$ is the deformation gradient tensor, $R_{e}$ is the rotation matrix and $J_{e}=\operatorname{det}\left(F_{e}\right)$. In view of different contributions to topological changes, the energy density function can be decomposed into two parts : $\mu\left\|F_{e}-R_{e}\right\|^{2}$ as deviatoric part and $\frac{\lambda}{2}\left(J_{e}-1\right)^{2}$ as isotropic part. However, $F_{e}$ is based on the spatial derivatives of displacement which leads to the inability of the constitutive model to compute singularity issues, such as discontinuities. We can adopt the concept of integral deformation matrix $\overline{F_{p}}$ in [7] to describe the local deformation which has a similar meaning to $F_{e}$ but is represented by the integration of displacement states and peridynamics truss structure. When the initial bond state and deformed bond between material point $x_{p}$ and $x_{p}{ }^{\prime}$ are $X=x_{p}-x_{p}{ }^{\prime}$ and $Y=y_{p}-y_{p}{ }^{\prime}$, the deformation matrix $\overline{F_{p}}$ shown in Equation 4 represents the average deformation status of point $x_{p} . H_{x_{p}}$ represents all family members. $w(Y)$ is the linear weight function. $\otimes$ is the dyadic product operator defined by Silling [20]. Thus an average deformed bond is calculated as $\bar{Y}=\overline{F_{i}} X$.

$$
\overline{F_{p}}=\left[\sum_{H_{x_{p}}} w(Y) Y \otimes X\right]\left[\sum_{H_{x_{p}}} w(Y) \otimes X\right]^{-1}
$$


With these concepts, Equation 3 is reformulated as the combination of the deviatoric and isotropic components :

$$
E^{s}=\sum_{H_{x_{p}}} w(Y)\left(\mu E^{d e v}+\frac{\lambda}{2} E^{i s o}\right)
$$

$E^{\text {dev }}=\left(\frac{|\bar{Y}|}{|X|}-1\right)^{2}$ describes deformed energy similar to a mass spring system but removes the influence from differing bond lengths in order to simulate material with the same stiffness. It involves the weighed deformed bond length. Similarly, $E^{\text {iso }}=\left(\frac{|Y|}{|X|}-1\right)^{2}$ represents single bond deformation energy.

Then the elastic force density function $T<x_{p}, x_{p}{ }^{\prime}>$ for the material point pair of $x_{p}$ and $x_{p}{ }^{\prime}$ is obtained through $\frac{\partial \psi}{\partial y_{p}}$, as:

$$
T<x_{p}, x_{p}{ }^{\prime}>=\frac{2 \mu w}{|X|^{2}}(\bar{Y}-|X| \operatorname{dir} \bar{Y})+\frac{\lambda w}{|X|^{2}}(Y-|X| \operatorname{dir} Y)
$$

where $\frac{2 \mu w}{|X|^{2}}(|\bar{Y}|-|X|) \operatorname{dir} \bar{Y}$ involves the deformed bonds in the whole neighbor, similar to sheer stress effects. $\frac{\lambda w}{|X|^{2}}(|Y|-|X|) \operatorname{dir} Y$ is equalled to the spring force between by $x_{p}$ and $x_{p}{ }^{\prime}$. The direction follows the current deformed bond. Using Equation 2 and Equation 6, the internal force of grid node $I$ for updating nodal momentum is:

$$
f_{I}^{I N T}=\sum m_{p} N_{I}\left(x_{p}\right)\left[\sum_{H_{x_{p}}} w(Y)\left(T<x_{p}, x_{p}{ }^{\prime}>-T<x_{p}{ }^{\prime}, x_{p}>\right) V\right]
$$

Compared to many existing MPM methods, our method avoids the singular value decomposition (SVD) which is used to extract the elastic deformation gradient. We only involve the current virtual bond state $Y$ for processing nodal internal forces, so the local step is fast. The standard SVD implementations can have a dramatic impact on performance [3]. Thus although it is not essential for performance to avoid the SVD, it is preferable not to implement the SVD. Also, as with our model, the advantages of avoiding SVD are obvious: we obtain a better stability for simulation with large time steps as in [7]; and reduce the complexity of the plasticity definition in the MPM framework.

\subsection{Plastic Model}

Many methods [27][35] take out part of the elastic deformation gradient tensor that exceeds the yield function and push it into the plastic deformation gradient calculation. Thanks to the construction of virtual bonds, the plasticity can be regarded as part of the bond's elastic extension and extracted within the yield criterion simply.

The plastic model is purely from deviatoric plastic flow theory [4]. Firstly, we reformulate our elastic model in Equation 6 in order to adapt it for modeling plasticity. $|\bar{Y}|-|X|$ and $|Y|-|X|$ are average and single bond extension. When 
the deformation is smooth enough under small neighbor horizon, we predict $|\bar{Y}|-|X| \approx|Y|-|X|$. Then we have:

$$
T<x_{p}, x_{p}{ }^{\prime}>=\frac{2 \mu w}{|X|^{2}}(|Y|-|X|) \operatorname{dir} \bar{Y}+\frac{\lambda w}{|X|^{2}}(|Y|-|X|) \operatorname{dir} Y
$$

Based on the plastic flow theory, the unified displacement is decomposed into isotropic and deviatoric parts, $e=(|Y|-|X|) /|X|=e^{i s o}+e^{\text {dev }}$. Plastic deformation $e^{p}$ is extracted from $e^{d e v}$. Incorporate the plastic extension $e^{p}$ into Equation 8:

$$
T<x_{p}, x_{p}{ }^{\prime}>=\frac{2 \mu w}{|X|}\left(e^{i s o}+e^{d e v}-e^{p}\right) \operatorname{dir} \bar{Y}+\frac{\lambda w}{|X|}\left(e^{i s o}+e^{d e v}-e^{p}\right) \operatorname{dir} Y
$$

The bond concept helps divide the deformation into elasticity and plasticity with the yield function as $f\left(E_{d e v}\right)$ :

$$
E_{\text {dev }}=\left(\frac{2 \mu w}{|X|}+\frac{\lambda w}{|X|}\right)\left(e^{\text {dev }}-e^{p}\right), f\left(E_{\text {dev }}\right)=\frac{\left(E_{\text {dev }}\right)^{2}}{2}-\psi_{p}
$$

where $\psi_{p}$ is a controllable plastic material parameter. We use $f\left(E_{d e v}\right)$ to decide if the current configuration enters the plastic regime. If $f\left(E_{\text {dev }}\right)<0$, the deformation is still within the elastic domain. If $f\left(E_{\text {dev }}\right)>0$, part of deformation occurred as plasticity. We project the deformation back to the yield surface and add a plastic increment $\Delta e^{p}$ to $e^{p}$ permanently as Equation 11.

$$
\Delta e^{p}=\frac{|X|}{(\lambda)}\left[E_{d e v}-\sqrt{2 \psi_{p}} \operatorname{sign}\left(E_{d e v}\right)\right]
$$

This model is still valid for elastic when $e^{p}$ varnishes in above equations. With the appropriate plastic material parameter, the plastic model can be a user-controllable constitutive model for simulating elasticity and elastoplasticity simultaneously under the MPM framework.

\section{$5 \quad$ Linear Viscoelastic Models}

Viscoelastic materials behave with elastic resistance to deformation similarly to elastic objects, while undergoing large strains and complex non-Newtonian fluid characteristics. Here the Eulerian background grid in MPM is still suitable for implicit integration, while the particle dynamics is represented by the modulus state which is arising from the linearized peridynamics theory by Silling [21]. Through superposing the small deformation on an existing large deformation in the configuration of last step, the internal force density function takes the small incremental bond extension to update the response of viscoelastic material step by step. In Silling's linearized peridynamics theory [21], to calculate the internal force density function at the next step, the deformed bond state at current step is regarded as the reference configuration. With this prerequisite, the elastic 
force density in Equation 6 needs to involve the information of the reference configuration.

Let $u$ be the small displacement field superposed on the current deformation field. Linearizing the function $T<x_{p}, x_{p}{ }^{\prime}>$ near current deformation field leads to :

$$
T<x_{p}, x_{p}{ }^{\prime}>=T^{0}<x_{p}, x_{p}{ }^{\prime}>+K<x_{p}>\cdot U<x_{p}>
$$

where $T^{0}<x_{p}, x_{p}{ }^{\prime}>$ is the deformed bond force density at the previous time step, $U<x_{p}>=\sum_{H_{x_{p}}} w(Y)(Y-X)$ is the average displacement near the point $x_{p} . K<x_{p}>=\nabla T\left(x_{p}, x_{p}{ }^{\prime}\right)$, is called the modulus state. It is the Fréchet derivative of the force density function with respect to the bond $Y^{\prime}$ which is between $x_{p}$ and one of its family particles $x_{p}{ }^{\prime \prime} . Y^{\prime}$ may not be the same bond as current bond $Y$. K replaces the stress strain in the previous sections to represent the deformation of material particles in linearized constitutive models. Detailed explanation can be found in Silling's work [21] [22].

Note the linearized equation, thus the internal force density function $F^{s}$ is rewritten as :

$$
\begin{aligned}
F^{s}\left(x_{p}\right)= & \int_{\Omega} \int_{\Omega}\left[T^{0}<x_{p}, x_{p}{ }^{\prime}>+K<x_{p}>\cdot U<x_{p}>\right)- \\
& \left.\left(T^{0}<x_{p}{ }^{\prime}, x_{p}>+K<x_{p}{ }^{\prime}>\cdot U<x_{p}^{\prime}>\right)\right] / \rho d H_{x_{p}}
\end{aligned}
$$

Based on the assumption of the equilibrated deformation as in [21], the reference configuration is assumed as equilibrated which indicates $\int_{H_{x_{p}}}\left(T^{0}<x_{p}, x_{p}{ }^{\prime}>\right.$ $\left.\left.-T^{0}<x_{p}{ }^{\prime}, x_{p},>\right)\right] / \rho d H_{x_{p}}+b\left(x_{p}\right)=0$.

The energy density function therefore is arranged leading to:

$$
\begin{aligned}
F^{s}\left(x_{p}\right) & =\int_{H_{x_{p}}} \int_{H_{x_{p}}}\left[K\left[x_{p}\right]<x_{p}{ }^{\prime}-x_{p}, x_{p}{ }^{\prime \prime}-x_{p}>\left(u\left(x_{p}{ }^{\prime \prime}\right)-u\left(x_{p}\right)\right)\right] / \rho d H_{x_{p}} d H_{x_{p}} \\
& -\int_{H_{x_{p}}} \int_{H_{x_{p}}{ }^{\prime}}\left[K\left[x_{p}{ }^{\prime}\right]<x_{p}-x_{p}{ }^{\prime}, x_{q}-x_{p}{ }^{\prime}>\left(u\left(x_{q}\right)-u\left(x_{p}{ }^{\prime}\right)\right)\right] / \rho d H_{x_{p}{ }^{\prime}} d H_{x_{p}}
\end{aligned}
$$

where $x_{p}{ }^{\prime}$ and $x_{p}{ }^{\prime \prime}$ are family particles of the point $x_{p}, x_{q}$ is the family particle of the point $x_{p}{ }^{\prime}$. This function not only involves the family particles of point $x_{p}$, but also the family particles $x_{q}$ of point $x_{p}{ }^{\prime}$. Thus the zone being influenced is fundamentally double than the horizon defined before. Fig 1 shows the indirect interaction between $x_{p}$ and $x_{q}$.

This linearization of the internal force density function is operated in the sense of a limit, under suitable restrictions on the smoothness of the deformation, motivated by Silling's work [21]. Through the linearization, the governing equation requires the current displacement and the modulus state, which are irrelevant to the original object shape, leading to structureless material effects. Therefore, the linear theory is naturally appropriate for colloidal materials, such as foam, cream and sponge because it is able to keep the response of elastic 


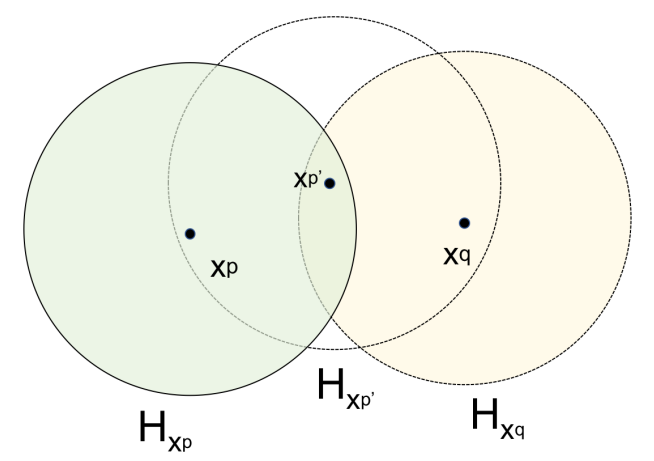

Fig. 1: Point $x_{p}$ interacts with $x_{q}$ even though they are outside each other's horizon because they are both within the horizon of intermediate points such as $x_{p}{ }^{\prime}$

material properties by utilizing the truss particle structures and to investigate the large topological changes experienced by processing the small incremental deformation based on the current large deformation field. Also, similar to classic peridynamics theory, the linear theory doesn't involve the deformation gradient, in other words, the crack propagation can be defined spontaneously by bond deactivation.

The viscoelastic model defined in [21] and [24] achieves the viscous elastic effects. It combines the strain energy density of the integral fluid constitutive models with the one energy density component which involves the deviatoric part of the deformation state (in the term $e-\frac{\vartheta}{3}$ ). Then energy density function is defined as:

$$
\begin{gathered}
E^{s}=\frac{\lambda \vartheta^{2}}{2}+\frac{\mu}{2} \sum_{H} w(|Y|)\left(e-\frac{\vartheta}{3}\right)^{2} V \\
\vartheta=\frac{3}{M} \sum_{H} w(|Y|)|Y| e V \\
M=\sum_{H} w(|Y|)|Y|^{2} V
\end{gathered}
$$

e is the extension of the bond, as defined in last section. $\vartheta$ represents the dilatation part of the bond extension. Thus the deviatoric part means the bond extension subtracts an isotropic expansion of the family particles. It includes not only shear, but also any deformation from the family particles other than isotropic expansion. Then the viscoelastic force density function $T\left\langle x_{p}, x_{p}{ }^{\prime}\right\rangle$ is written as:

$$
T<x_{p}, x_{p}^{\prime}>=\left(\frac{3 \lambda}{M}-\frac{\mu}{3}\right) w(|Y|)|Y| \vartheta M+\mu w(|Y|) \operatorname{edir} Y
$$


Here we define two bonds, $Y_{1}=x_{p}^{\prime}-x_{p}$ and $Y_{2}=x_{p}^{\prime \prime}-x_{p}$ which are incorporated in the modulus state $\mathrm{K}$. Thus $\mathrm{K}$ is written as:

$$
\begin{gathered}
K<x_{p}{ }^{\prime}-x_{p}, x_{p}{ }^{\prime \prime}-x_{p}>=\left(\frac{9 \lambda}{M^{2}}-\frac{\mu}{M}\right) w\left(\left|Y_{1}\right|\right) w\left(\left|Y_{2}\right|\right) Y_{1} \otimes Y_{2}+\gamma\left(Y_{1}\right) \Delta\left(Y_{2}-Y_{1}\right) \\
\gamma(Y)=\lambda w(|Y|)(\operatorname{dir} Y \otimes \operatorname{dir} Y)
\end{gathered}
$$

$\Delta$ denotes the Dirac delta function in the simulation field. With the modulus state and current deformation field, the force density function is obtained.

\section{Fracture}

Crack simulation is a bottleneck of the MPM [14]. To processing the discontinuities at the interfaces, special treatments for creating cracks and partitioning fracture fragments into multigrid and multiple velocity fields are approaches [8]. When an excessive number of cracking interfaces are involved, the computation of multiple grid transferring can be very expensive. Additionally, the strategy to duplicate a grid is limited because during simulation small fragments are numerous and randomly generated so it is hard to duplicate a grid for each crack interface.

The dynamics with discontinuities is straightforward to compute in our MPM framework. If we simply remove over-deformed bonds like [4], it leads to numerous small fragments in the deformed area rather than several crack lines after collision happens. We now propose generating a crack cut by the fracture plane based on analyses of single point and global deformation status in the gird cell.

Firstly, we define the fracture criterion by removing the plastic displacement as:

$$
l=\frac{e-e^{p}}{|X|\left(1+p_{\text {inactive }}\right)}
$$

where $p_{\text {inactive }}$ is the percentage of broken bonds in total bonds in one grid cell where the material point stays.

After screening the material points whose $l$ exceeds the threshold, we use cluster method to sort these points into several deformed areas based on position and normal. For each area, we calculate the central point and the largest deformed bond. The next step is to use the central point position and the bond direction as normal to construct the fracture plane for each area. Any bond intersected by the fracture plane will be removed. That is how crack line occurs. With $p_{\text {inactive }}$, this method can effectively reduce the number of small fracture pieces. Because if one grid has too many broken bonds, the active bonds in this grid cell are less likely to be removed.

In the MPM, grid cell size decides the resolution of whole simulation. This also works for crack dynamics. In experiments, we transfer particle velocity to three grid cells (illustrated in Fig.2) in any direction to get stable, smooth results. When two sides of crack line are within this range, they will share additional 


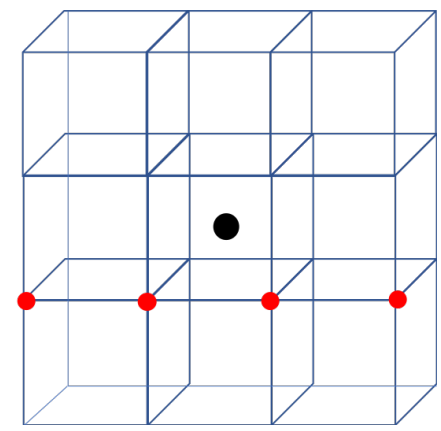

Fig. 2: For MPM transfer process, the information of one particle is transferred to neighbor 9 cell in 2D dimension.

fragment information through transfer. Therefore the using of one grid leads to "fractures sticking to each other" effects. We alleviate this problem by applying two-time integration methods: material points on the crack surface are updated by its own bond forces; other material points which don't have any broken bonds (in the fragmented inner parts) are updated by grids as normal. Thus we avoid the mixing of altered crack fragments.

\section{Result and Discussion}

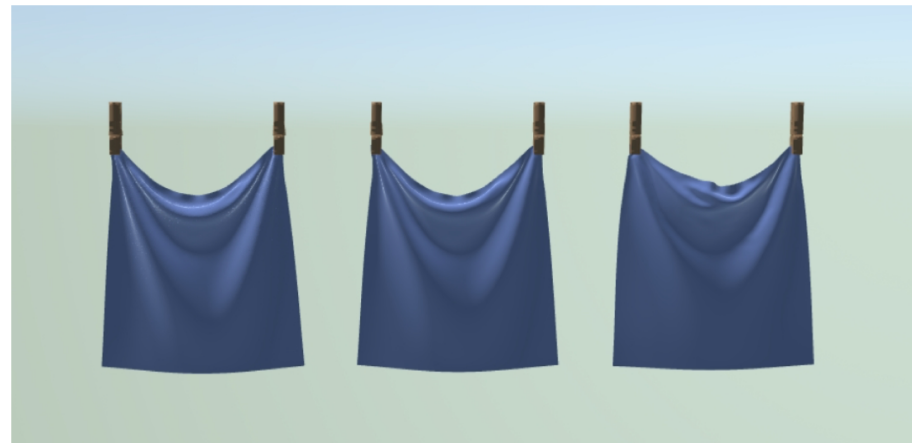

Fig. 3: The moving cloth with different material parameters show differing bending stiffness. From left to right: $\mu=2 \times 10^{4}, \mu=1.5 \times 10^{5}, \mu=1 \times 10^{6}$.

We implemented the proposed methods and tested examples in this section. All examples are produced in Houdini software, including the material point discretization, dynamics processing, vorinoi fracture generation and rendering of the simulation. The Houdini Development Kit (HDK) tool is utilized to create customized dynamic nodes for the constitutive models. 

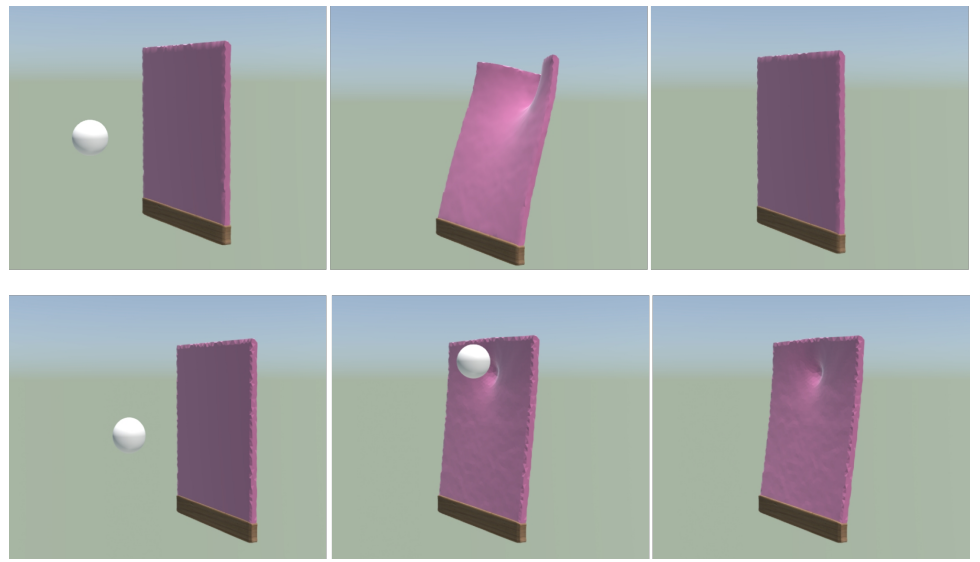

Fig. 4: Throwing a ball against elastic and plastic boards shows visibly different results. In the first row, the elastic board can recover to the initial shape after the collision. The second row shows that plastic board keeps the deformed topology.

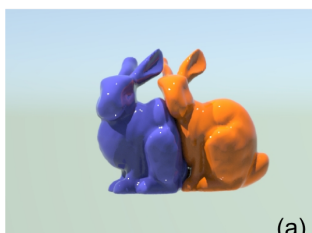

(a)

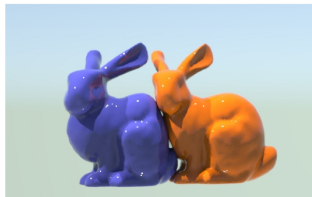

(c)

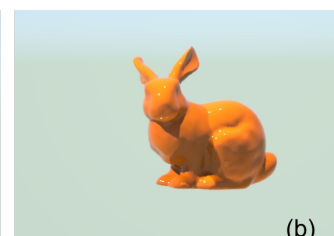

(b)

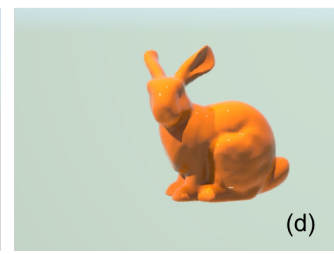

Fig. 5: The collision between two identical rabbits with different materials. This example demonstrates the different deformation of elastic rabbits (in the first row) and plastic rabbits (in the second row). Collision happens in (a) and (c). The elastic rabbits are able to recover as in (b). The plastic rabbits deformed afterwards in (d). 


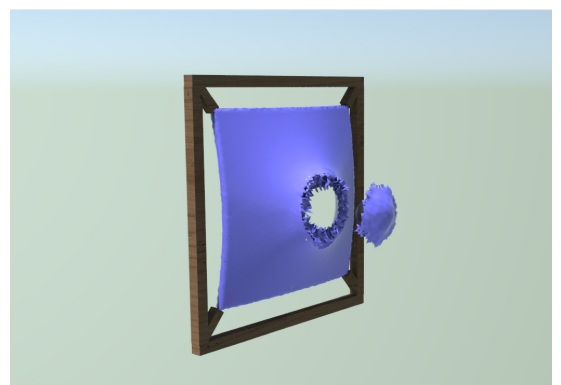

Fig. 6: Simulation of a plastic wall when collided by a sphere.

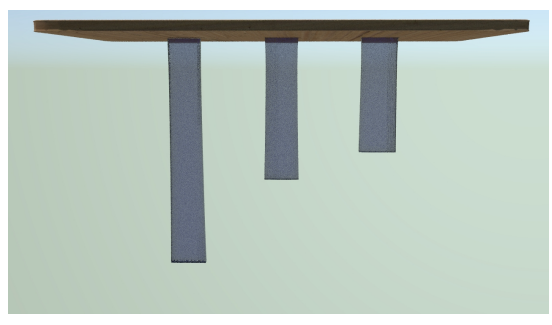

Fig. 7: Stretch beams with different material parameters. From left to right, bending stiffness $\mu$ are: $0,50,500$.

Implementation. In this paper, three different modeling geometry types are used.

Mesh based geometry: With the given input surface, we construct mesh as the surface and initialize several layers of particles underneath the surface.

Particle based geometry: For specific examples, such as the viscous elastic material, we represent the initial object shape with the unstructured particle, due to its simplicity.
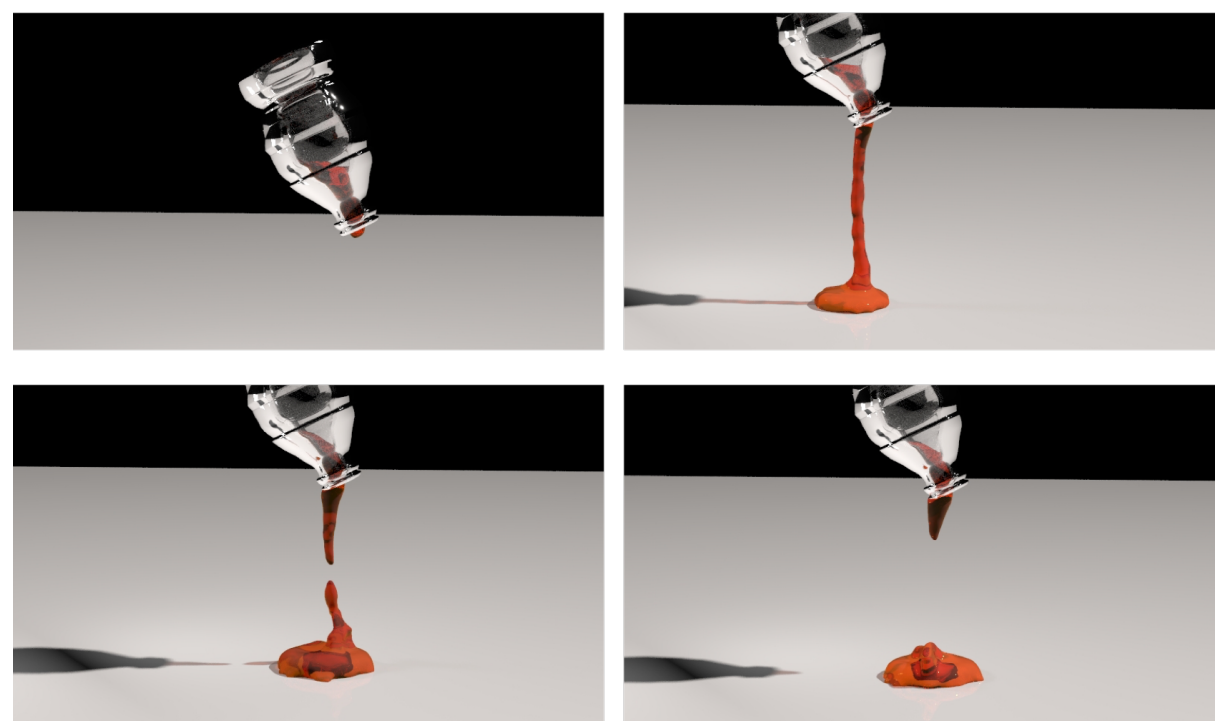

Fig. 8: Linear peridynamics theory is used for modeling viscoelastic fluids, such as honey.

Voronoi cell geometry: Houdini designs the voronoi cell particularly for fracture or cracks scenes. Each voronoi cell is a random polygon and its center point 

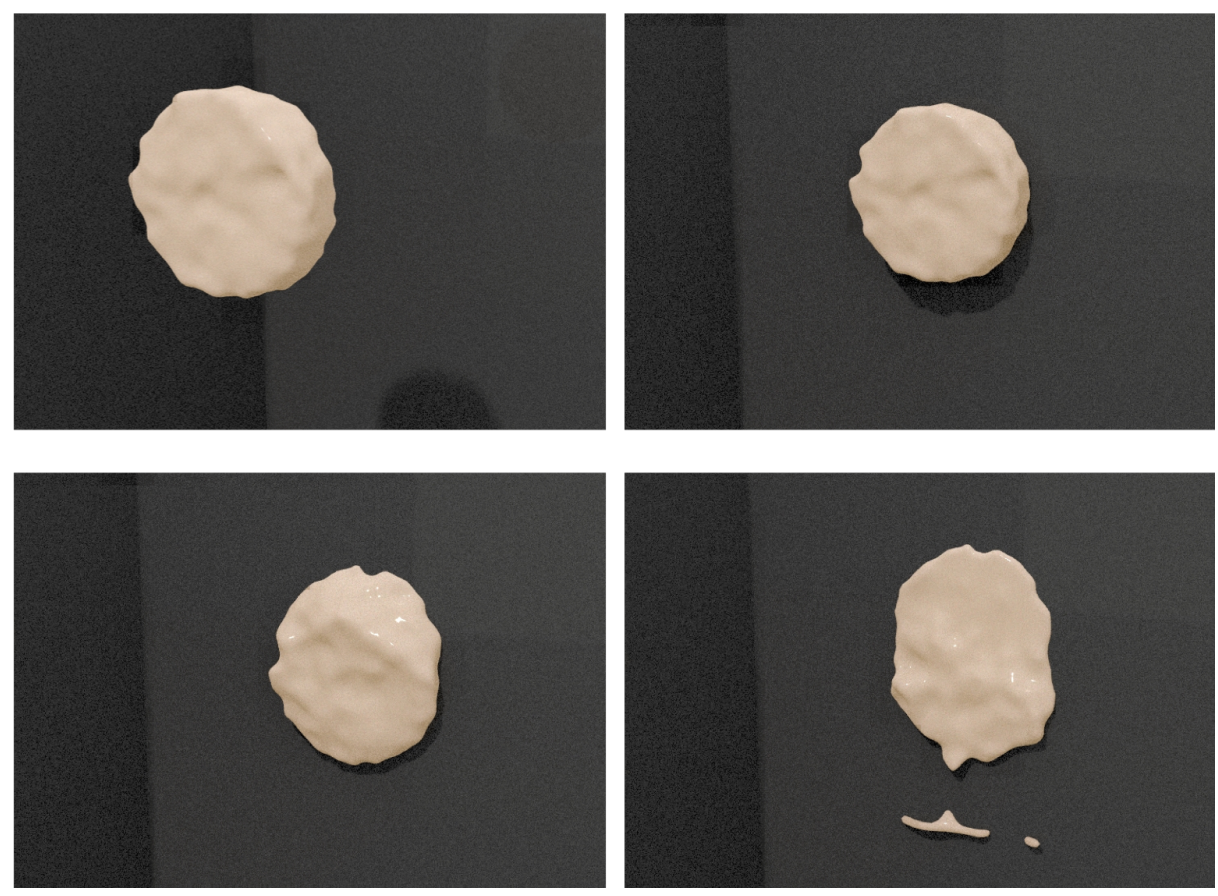

Fig. 9: A pie of whipped cream is thrown to the wall to show the effects of viscoelasticity.

is used as the material points. The bond represents the connection between neighboring voronoi cells.

Table 1 lists the modeling types, parameter settings and the performance data for all the examples presented in the paper. Ghost particles are added out of the object surface to guarantee that each material point has similar family density in the initial steps.

Model validation. We use several examples with different material properties to evaluate our method. Fig. 3 shows the examples of garment anchored by clothes pegs. With varied bending and stiffness parameters $\mu$, the experiments present the realistic and fine wrinkles. Fig. 4 shows the comparison of elastic board and plastic board collided by a ball. Our model can create correct behaviors. With complex topology objects, this method still works as in Fig. 5. Fig. 6 shows the simulation of ductile plastic fracture. Fig. 7 demonstrates the stretching beams deformation with different material stiffness. Fig. 8 shows that honey flows from a glass bottle and drops on the floor. Linear peridynamics model is able to represent the viscoelastic fluids situation. Fig. 9 shows that when whipped cream is thrown to the wall, it flows like dense fluid, whilst slightly retaining the original shape. 
Table 1: modeling information for all examples

\begin{tabular}{|c|c|c|c|c|c|c|c|}
\hline & Type & $\begin{array}{c}\text { Grid cell } \\
\text { size }\end{array}$ & $\lambda(\mathrm{MPa})$ & $\mu(\mathrm{MPa})$ & $\phi_{\mathrm{p}}$ & $\begin{array}{c}\text { Fracture } \\
\text { threshold }\end{array}$ & $\Delta \mathrm{t}(\mathrm{ms})$ \\
\hline Cloth & Mesh & 0.005 & $1 \times 10^{6}$ & $2 \times 10^{4}, 1 \times 10^{5}, 1.5 \times 10^{5}$ & $1 \times 10^{25}$ & $1 \times 10^{25}$ & 0.1 \\
\hline Elastic board & Mesh & 0.02 & $3 \times 10^{6}$ & $1.5 \times 10^{5}$ & $1 \times 10^{25}$ & $1 \times 10^{25}$ & 0.02 \\
\hline Plastic board & Mesh & 0.02 & $5 \times 10^{6}$ & $1.5 \times 10^{5}$ & 100 & $1 \times 10^{25}$ & 0.0001 \\
\hline Elastic bunny & Particles & 0.01 & $1 \times 10^{5}$ & $1 \times 10^{5}$ & $1 \times 10^{25}$ & $1 \times 10^{25}$ & 0.1 \\
\hline Plastic bunny & Particles & 0.01 & $1 \times 10^{6}$ & $3 \times 10^{5}$ & 30 & $1 \times 10^{25}$ & 0.01 \\
\hline Broken board & Voronoi & 0.005 & $1 \times 10^{6}$ & $5 \times 10^{4}$ & 500 & 0.05 & 0.001 \\
\hline Stretching beam & Mesh & 0.05 & 500 & $0,50,500$ & $1 \times 10^{25}$ & $1 \times 10^{25}$ & 100 \\
\hline Honey & Particles & 0.05 & 500 & $1 \times 10^{2}$ & - & 0.0304 & 10 \\
\hline Cream pie & Particles & 0.04 & $5 \times 10^{3}$ & $5 \times 10^{2}$ & - & 0.035 & 10 \\
\hline
\end{tabular}

\section{Conclusion}

We present the integral-based Material Point Method using peridynamics particle structure. This method integrates the deformation displacement of particles to compute the internal forces for grid nodes, which avoids the instability and complex implementation issues existing in the current MPM. This paper demonstrates various elastic deformation and plastic deformation scenarios. The linearized peridynamics theory is introduced in the paper to simulate general materials. Fracture can be modeled robustly without any singularity issues. Additionally, our method presents a novel integral-based view for multi-scale multimaterial modeling and fractures modeling, which has potential to inspire future research in the field.

Limitations and Future work. This paper presents numerous examples with elastic and plastic material. However, there is still much work to be achieved. Firstly, we represent fracture with Houdini voronoi structure which is represented by polygon with random number of vertexes. It is unable to generate the arbitrary fracture shape. The resolution is limited to the number and size of voronoi cells. Incorporating tetrahedron structure for a embedded geometry modeling is the future option for cooking detailed cracking interfaces. Secondly, the linearized peridynamics exhibits potential to simulate multi-scale materials using the similar viscoelastic models, such foam which is the combination of bubble and solids. We will explore more applications in future. Finally, we use explicit integration for its straightforwardness in HDK. It requires very small substeps for dealing with huge displacement. Additionally, the current study implements projective dynamics implicit integration method for fast simulation. It can obtain stable and robust results under large substeps. Our future work will focus on 
addressing above limitations and obtaining versatile and realistic elastoplastic performance.

\section{References}

1. Böttcher, G.: Haptic Interaction with Deformable Objects: Modelling VR Systems for Textiles. Springer Science \& Business Media (2011)

2. Bottcher, G., Allerkamp, D., Wolter, F.E.: Virtual reality systems modelling haptic two-finger contact with deformable physical surfaces. In: 2007 International Conference on Cyberworlds (CW'07). pp. 292-299. IEEE (2007)

3. Chao, I., Pinkall, U., Sanan, P., Schröder, P.: A simple geometric model for elastic deformations. ACM transactions on graphics (TOG) 29(4), 38 (2010)

4. Chen, W., Zhu, F., Zhao, J., Li, S., Wang, G.: Peridynamics-based fracture animation for elastoplastic solids. In: Computer Graphics Forum. vol. 37, pp. 112-124. Wiley Online Library (2018)

5. Gao, M., Tampubolon, A.P., Jiang, C., Sifakis, E.: An adaptive generalized interpolation material point method for simulating elastoplastic materials. ACM Transactions on Graphics (TOG) 36(6), 223 (2017)

6. Gerszewski, D., Bhattacharya, H., Bargteil, A.W.: A point-based method for animating elastoplastic solids. In: Proceedings of the 2009 ACM SIGGRAPH/Eurographics Symposium on Computer Animation. pp. 133-138. ACM (2009)

7. He, X., Wang, H., Wu, E.: Projective peridynamics for modeling versatile elastoplastic materials. IEEE transactions on visualization and computer graphics $\mathbf{2 4}(9)$, 2589-2599 (2018)

8. Homel, M.A., Herbold, E.B.: Field-gradient partitioning for fracture and frictional contact in the material point method. International Journal for Numerical Methods in Engineering 109(7), 1013-1044 (2017)

9. Jiang, C., Gast, T., Teran, J.: Anisotropic elastoplasticity for cloth, knit and hair frictional contact. ACM Transactions on Graphics (TOG) 36(4), 152 (2017)

10. Jiang, C., Schroeder, C., Selle, A., Teran, J., Stomakhin, A.: The affine particlein-cell method. ACM Transactions on Graphics (TOG) 34(4), 51 (2015)

11. Jiang, C., Schroeder, C., Teran, J., Stomakhin, A., Selle, A.: The material point method for simulating continuum materials. In: ACM SIGGRAPH 2016 Courses. p. 24. ACM (2016)

12. Levin, D.I., Litven, J., Jones, G.L., Sueda, S., Pai, D.K.: Eulerian solid simulation with contact. ACM Transactions on Graphics (TOG) 30(4), 36 (2011)

13. Levine, J.A., Bargteil, A.W., Corsi, C., Tessendorf, J., Geist, R.: A peridynamic perspective on spring-mass fracture. In: Proceedings of the ACM SIGGRAPH/Eurographics Symposium on Computer Animation. pp. 47-55. Eurographics Association (2014)

14. Liang, Y., Benedek, T., Zhang, X., Liu, Y.: Material point method with enriched shape function for crack problems. Computer Methods in Applied Mechanics and Engineering 322, 541-562 (2017)

15. Lyu, Y., Zhang, J., Chang, J., Guo, S., Zhang, J.J.: Integrating peridynamics with material point method for elastoplastic material modeling. In: Computer Graphics International Conference. pp. 228-239. Springer (2019)

16. Moutsanidis, G., Kamensky, D., Zhang, D.Z., Bazilevs, Y., Long, C.C.: Modeling strong discontinuities in the material point method using a single velocity field. Computer Methods in Applied Mechanics and Engineering 345, 584-601 (2019) 
17. O'brien, J.F., Bargteil, A.W., Hodgins, J.K.: Graphical modeling and animation of ductile fracture. In: ACM transactions on graphics (TOG). vol. 21, pp. 291-294. ACM (2002)

18. Ram, D., Gast, T., Jiang, C., Schroeder, C., Stomakhin, A., Teran, J., Kavehpour, P.: A material point method for viscoelastic fluids, foams and sponges. In: Proceedings of the 14th ACM SIGGRAPH/Eurographics Symposium on Computer Animation. pp. 157-163. ACM (2015)

19. Salsedo, F., Fontana, M., Tarri, F., Ruffaldi, E., Bergamasco, M., MagnenatThalmann, N., Volino, P., Bonanni, U., Brady, A., Summers, I., et al.: Architectural design of the haptex system. In: submitted to the Proceedings of this Conference (2005)

20. Silling, S.A.: Reformulation of elasticity theory for discontinuities and long-range forces. Journal of the Mechanics and Physics of Solids 48(1), 175-209 (2000)

21. Silling, S.A.: Linearized theory of peridynamic states. Journal of Elasticity 99(1), 85-111 (2010)

22. Silling, S.A.: A coarsening method for linear peridynamics. International Journal for Multiscale Computational Engineering 9(6) (2011)

23. Silling, S.A., Askari, A.: Peridynamic model for fatigue cracking. SAND2014-18590. Albuquerque: Sandia National Laboratories (2014)

24. Silling, S.A., Epton, M., Weckner, O., Xu, J., Askari, E.: Peridynamic states and constitutive modeling. Journal of Elasticity 88(2), 151-184 (2007)

25. Silling, S.A., Askari, A.: Practical peridynamics. Tech. rep., Sandia National Lab.(SNL-NM), Albuquerque, NM (United States) (2014)

26. Stomakhin, A., Howes, R., Schroeder, C., Teran, J.M.: Energetically consistent invertible elasticity. In: Proceedings of the ACM SIGGRAPH/Eurographics Symposium on Computer Animation. pp. 25-32. Eurographics Association (2012)

27. Stomakhin, A., Schroeder, C., Chai, L., Teran, J., Selle, A.: A material point method for snow simulation. ACM Transactions on Graphics (TOG) 32(4), 102 (2013)

28. Stomakhin, A., Teran, J., Selle, A.: Augmented material point method for simulating phase changes and varied materials (Jul 2 2015), uS Patent App. 14/323,798

29. Sulsky, D., Chen, Z., Schreyer, H.L.: A particle method for history-dependent materials. Computer methods in applied mechanics and engineering 118(1-2), 179-196 (1994)

30. Sulsky, D., Zhou, S.J., Schreyer, H.L.: Application of a particle-in-cell method to solid mechanics. Computer physics communications 87(1-2), 236-252 (1995)

31. Tampubolon, A.P., Gast, T., Klár, G., Fu, C., Teran, J., Jiang, C., Museth, K.: Multi-species simulation of porous sand and water mixtures. ACM Transactions on Graphics (TOG) 36(4), 105 (2017)

32. Terzopoulos, D., Fleischer, K.: Modeling inelastic deformation: viscolelasticity, plasticity, fracture. In: ACM Siggraph Computer Graphics. vol. 22, pp. 269-278. ACM (1988)

33. Terzopoulos, D., Platt, J., Barr, A., Fleischer, K.: Elastically deformable models. ACM Siggraph Computer Graphics 21(4), 205-214 (1987)

34. Xu, L., He, X., Chen, W., Li, S., Wang, G.: Reformulating hyperelastic materials with peridynamic modeling. In: Computer Graphics Forum. vol. 37, pp. 121-130. Wiley Online Library (2018)

35. Zhu, B., Lee, M., Quigley, E., Fedkiw, R.: Codimensional non-newtonian fluids. ACM Transactions on Graphics (TOG) 34(4), 115 (2015) 